\title{
Recent observations on drugs and human fertility
}

\author{
Paul Turner
}

Department of Clinical Pharmacology, St. Bartholomew's Hospital, London EC1A 7BE, UK.

\begin{abstract}
Summary: The transmembrane migration method has permitted quantification of the effect of drugs on human sperm motility. Calcium ionophores and calcium antagonists have paradoxical effects on motility depending on the stage of sperm maturity. Drugs with membrane stabilizing properties inhibit sperm motility. Propranolol, for example, has been shown to have contraceptive activity when administered intra-vaginally. Pharmacokinetic studies have shown that the systemic availability of vaginally administered propranolol is markedly greater than after oral administration. Seminal fluid concentrations of propranolol after oral administration are similar to those in plasma, but cervicovaginal mucus concentrations are much higher, and residence times much longer. The clinical consequences of these observations await elucidation.
\end{abstract}

There is increasing interest in the clinical pharmacology of human sperm motility, for seveal reasons. Firstly, the development of in vitro fertilization has necessitated research into factors influencing the motility and ovum-penetrating properties of sperms. Secondly, the demonstration that drugs taken by male patients may influence their fertility by interfering with spermatogenesis or sperm function has increased the awareness of the importance of this subject. Thirdly, and related to this, there is the possibility that drugs taken orally or otherwise by a woman, or inserted intravaginally, might influence the motility of sperms deposited into her vagina during intercourse. Finally, there is widespread interest in finding alternatives to animal models to study the pharmacological properties of drugs, among which are in vitro systems using isolated human cells and tissues. It may be that human sperms could provide such a model.

Sperm motility has been observed since the earliest use of the microscope by Leeuwenhoek. Several techniques are now available to quantify sperm motility, including multiple exposure photography, laser Doppler spectroscopy and turbidometry, ${ }^{1}$ but they are complicated and timeconsuming, and do not lend themselves to pharma-

Correspondence: Professor P. Turner, B.Sc., M.D., F.R.C.P.

Received: 15 March 1988.

Based on a lecture given to the European Medical Research Group on 15 March 1988. cological studies involving dose-response relationships on single ejaculates of seminal fluid.

The transmembrane migration method for measuring drug effects on sperm motility

The transmembrane migration method (TMM) was specifically designed to permit the determination of inhibitory or facilitatory concentrations of drugs on human sperm motility, from dose response curves in duplicate or single ejaculates of seminal fluid. ${ }^{2}$ In principle it involves measurement of the proportion of sperms moving across the 5 micron pores of a Nucleopore membrane from an aliquot of semen into a buffer solution. In the original method, different drug concentrations were added only to the chamber containing the seminal fluid, but recently a modified version has been introduced ${ }^{3}$ which involves adding drug, in addition, to the buffer solution into which the sperms penetrate, and which produces EC50 concentrations which are more similar to those obtained by other methods.

Using the TMM, sperm immobilizing potencies of several groups of drugs have been determined, including antidepressants, antiarrhythmics, antihistamines, cannabis, local anaesthetics, neuroleptics, opiates, vaginal contraceptives, calcium antagonists and beta-adrenocepter blocking agents. ${ }^{1}$ These studies have demonstrated that drugs immobilize human sperms by acting on the cell membrane. Lipid solubility, which determines the absorption of drugs into the cell membrane, correlates with the sperm immobilizing potency. ${ }^{4}$ Drugs that inhibit

(C) The Fellowship of Postgraduate Medicine, 1988 
mitochondrial activity, such as cyanide, and microtubular function, such as colchicine, do not inhibit sperm motility. ${ }^{5,6}$ For these reasons, human sperm motility has been s.iggested as a potential model for the study of membrane stabilizing drugs. ${ }^{7,8}$ It may be that other ciliated or flagellated organisms could be used, for it has been shown that propranolol inhibits the motility of the flagellate protozoa Giardia lamblia and Trichomonas vaginalis. ${ }^{9}$

\section{Calcium ions and sperm motility}

The effects of calcium ions on sperm motility are paradoxical, depending upon the state of maturity of the sperms ${ }^{10}$ (Table I). Although evidence in human epididymal sperms is lacking, studies on several other mammalian species, including bull, mouse and hamster, have shown that calcium ions increase the motility of immotile sperms collected from the epididymis. Furthermore, there is evidence for a motility-promoting calcium-binding calmodulin-like protein in the sperm membrane at this stage of development.

Paradoxically, however, there is now substantial evidence that calcium ions inhibit the motility of ejaculated human sperms. Calcium chelators such as EGTA and EDTA as well as calcium antagonists such as diltiazem, flunarizine and verapamil stimulate sperm motility in ejaculated human semen. On the other hand, the calcium ionophore A23187, which increases intracellular calcium concentrations, inhibits human, dog and sea-urchin sperm motility. A protein that inhibits calcium uptake by ejaculated sperm has been purified from bovine seminal plasma, probably produced by the seminal vesicle.

Finally during their passage through the female genital tract, sperms are separated from seminal plasma and undergo capacitation. Calcium ions trigger the acrosome reaction, a calcium ionophore

Table I The paradoxical relationship between calcium and sperm motility

\begin{tabular}{lll}
\hline \multicolumn{1}{c}{$\begin{array}{c}\text { Stage of } \\
\text { maturation }\end{array}$} & \multicolumn{1}{c}{$\begin{array}{c}\text { Influence of } \\
\text { calcium }\end{array}$} & \multicolumn{1}{c}{$\begin{array}{c}\text { Transport } \\
\text { factors }\end{array}$} \\
\hline $\begin{array}{l}\text { Storage in } \\
\text { epididymis }\end{array}$ & $\begin{array}{l}\text { Promotes } \\
\text { motility }\end{array}$ & $\begin{array}{l}\text { Calmodulin-like } \\
\text { protein in sperm } \\
\text { tail }\end{array}$ \\
Ejaculation & $\begin{array}{l}\text { Inhibits } \\
\text { motility }\end{array}$ & $\begin{array}{l}\text { Calcium-transport } \\
\text { inhibitory } \\
\text { protein bound to } \\
\text { sperm membrane }\end{array}$ \\
& & $\begin{array}{l}\text { Removal of } \\
\text { calcium-transport } \\
\text { inhibitor }\end{array}$ \\
\hline
\end{tabular}

Based on Hong et al. ${ }^{10}$ inducing a premature acrosome reaction in uncapacitated sperms. ${ }^{10}$

It is evident that calcium ion concentration has a profound influence on motility of sperms according to their stage of maturity. Its relevance in procedures such as in vitro fertilization requires careful

\section{Caffeine and other xanthines}

Caffeine increases the motility of human sperms and also antagonizes the inhibitory effect of other drugs such as propranolol and procaine. ${ }^{11}$ The motility-enhancing activity of other xanthines such as isobutyl methylxanthine (IBMX) appears to correlate with their phosphodiesterase-inhibiting activity. ${ }^{12}$ Their safety in respect of teratogenic or sperm-injuring potential has not been established, however, and it would be premature to recommend their use to enhance sperm fertilizing ability at the present time.

\section{Propranolol, contraception and genital fluid concentration}

The TMM demonstrated that propranolol was a potent inhibitor of sperm motility in vitro, and that this activity appeared to reside in its membranestabilizing dextro-isomer. ${ }^{11}$

Zipper, noting an earlier report that propranolol, chlorpromazine and phenoxybenzamine inhibited sperm motility in vitro, ${ }^{13}$ carried out a study to evaluate the efficacy and tolerability of propranolol as a vaginal contraceptive. He found ${ }^{14}$ that an $80 \mathrm{mg}$ tablet of propranolol inserted into the vagina nightly from the last day of menstruation until the first day of the next menstrual period, without any other method of contraception, was associated with a one year life table pregnancy rate of $3.4 / 100$ women, and a Pearl index of 3.9/100 women years. No major adverse effects were encountered. A subgroup of these women were asked to insert the propranolol tablet intravaginally at $2100 \mathrm{~h}$ and have intercourse at about $0700 \mathrm{~h}$ the following morning. A post-coital test carried out 3 hours after intercourse did not show any motile sperms in the endocervical samples.

While it is probable that the direct sperm immobilizing action of propranolol demonstrated in the TMM studies accounts for its contraceptive action, the possibility of systemic action cannot be excluded. The systemic bioavailability of propranolol after vaginal administration is much greater than oral administration, ${ }^{15}$ not unexpectedly because the venous drainage of the vascular vaginal wall is directly into the inferior vena cava, so bypassing the hepatic circulation and avoiding the 
extensive first-pass metabolism which follows oral administration of propranolol. This must be remembered if the vaginal route is used as an alternative to oral in patients unable to swallow propranolol. ${ }^{16}$

The observation of a contraceptive action of propranolol administered by the vaginal route raises the question as to whether its oral use in a male could result in seminal fluid concentrations likely to inhibit his sperm motility, or whether oral ingestion by women could result in cervico-vaginal mucus concentrations which might inhibit the motility of their partners' sperms. Clinical pharmacological studies have shown that seminal fluid concentrations of propranolol after oral administration are similar to those in plasma, do not show any evidence of accumulation, and are unlikely to reach sperm-immobilizing levels after therapeutic doses. ${ }^{17}$ In females, however, cervico-vaginal mucus concentrations of propranolol after single oral doses show marked and extensive accumulation extending beyond $24 \mathrm{~h}$ after oral administration, when plasma levels are not measurable beyond 8$12 \mathrm{h.}^{18}$ This is, perhaps, not surprising, because the basic drug propranolol would be expected to be trapped in the acidic medium of the vagina, and to

\section{References}

1. Hong, C.Y. Pharmacological studies on human sperm motility. Trends Pharmacol Sci 1985, 6: 62-63.

2. Hong, C.Y., Chaput De Saintonge, D.M. \& Turner, P. A simple method to measure drug effects on human sperm motility. Br J Clin Pharmacol 1981, 11: 385-387.

3. Raoof, N.T., Pearson, R.M. \& Turner, P. A modified transmembrane migration method for measuring the effect of drugs on sperm motility. Br J Clin Pharmacol 1987, 24: 319-321.

4. Hong, C.Y. \& Turner, P. Influence of lipid solubility on the sperm immobilising effect of beta-adrenoceptor blocking drugs. $\mathrm{Br} J$ Clin Pharmacol 1982, 14: 269-272.

5. Hong, C.Y., Chiang, B.N. \& Wei, Y.H. Mitochondrial respiration inhibitors and human sperm motility: implication in the future development of spermicides. Br J Clin Pharmacol 1983, 16: 487-490.

6. Hong, C.Y, Chiang, B.N., Ku, J. \& Wu, P. Screening the in vitro sperm immobilising effect of some anticancer drugs. Human Toxicol 1985, 4: 461-464.

7. Turner, P. Isolated human cellular models in clinical pharmacological studies. In: Turner, P. (ed) Animals in Scientific Research: an Effective Substitute for Man? Macmillan, London, 1983, pp. 91-96.

8. Hong, C.Y., Wu, P., Sheih, C.C. \& Chiang, B.N. Membrane stabilising activity and inhibition of human sperm motility. Lancet 1986, ii: 402.

9. Farthing, M.J.G., Inge, P.M.G. \& Pearson, R.M. Effect of D-propranolol on growth and motility of flagellate protozoa. J. Antimicrob Chemother 1987, 20: 519-522. bind extensively to the glycoproteins of the vaginal mucus. Vaginal $\mathrm{pH}$ and mucus production vary, however, during the menstrual cycle and with age, and the clinical implications of these preliminary observations await further investigation and evaluation.

\section{Conclusion}

The pharmacokinetics of drugs into and out of genital compartments has been a neglected area of clinical pharmacology. It is evident, however, that drug penetration into, and residence within genital fluids may have important therapeutic and toxicological implications as well as an influence on fertility. Better understanding of the pharmacological basis of sperm motility should lead to a more rational approach to the design and performance of in vitro fertilization.

\section{Acknowledgements}

Many of the studies reviewed in the paper were supported by the Lawson Tait Medical and Scientific Research Trust, the Peel Medical Research Trust and Family Health International.

10. Hong, C.Y., Chiang, B.N. \& Turner, P. Calcium ion is the key regulator of human sperm function. Lancet 1984, ii: $1449-1451$.

11. Hong, C.Y., Chaput De Saintonge, D.M. \& Turner, $P$. The inhibitory action of procaine, $(+)$ propranolol and $( \pm)$ propranolol on human sperm motility: antagonism by caffeine. Br J Clin Pharmacol 1981, 12: 751-753.

12. Jiang, C.S., Kilfeather, S.A., Pearson, R.M. \& Turner, P. The stimulatory effects of caffeine, theophylline, lysine-theophylline and 3-isobutyl-1-methylxanthine on human sperm motility. Br J Clin Pharmacol 1984, 18: $258-262$.

13. Peterson, R.N. \& Freund, M. The inhibition of the motility of human spermatozoa by various pharmacologic agents. Biol Reprod 1975, 13: 552-556.

14. Zipper, J., Wheeler, R.G., Potts, D.M. \& Rivera, M. Propranolol as a novel, effective spermicide: preliminary findings. $\mathrm{Br}$ Med $J$ 1983, 287: 1245-1246.

15. Patel, L.G., Warrington, S.J. \& Pearson, R.M. Propranolol concentrations in plasma after insertion into the vagina. $B r$ Med J 1983, 287: 1247-1248.

16. Smith, R.C. \& Berge, M. Systemic absorption after intravaginal propranolol. Lancet 1985, i: 112.

17. Mahajan, P., Grech, E.D., Pearson, R.M., Ridgeway, E.J. \& Turner, P. Propranolol concentrations in blood serum, seminal plasma and saliva in man after a single oral dose. Br J Clin Pharmacol 1984, 18: 849-852.

18. Pearson, R.M., Ridgway, E.J., Johnston, A. \& Fadukul, J. Concentration of D-propranolol in cervico-vaginal mucus: targeting of a novel spermicide. Adv Contracept 1985, 1: 103-108. 